\title{
Glutathione peroxidase-2 protects from allergen-induced airway inflammation
} in mice

\author{
A.M. Dittrich**\#,f, H-A. Meyer*,f, M. Krokowski*, D. Quarcoo*, B. Ahrens*, \\ S.M. Kube ", M. Witzenrath ${ }^{\oplus}$, R.S. Esworthy ${ }^{+}$, F-F. Chu ${ }^{+}$and E. Hamelmann*,
}

ABSTRACT: The aim of the present study was to identify and validate the biological significance of new genes/proteins involved in the development of allergic airway disease in a murine asthma model.

Gene microarrays were used to identify genes with at least a two-fold increase in gene expression in lungs of two separate mouse strains with high and low allergic susceptibility. Validation of mRNA data was obtained by western blotting and immunohistochemistry, followed by functional analysis of one of the identified genes in mice with targeted disruption of specific gene expression.

Expression of two antioxidant enzymes, glutathione peroxidase-2 (GPX2) and glutathione Stransferase omega (GSTO) 1-1 was increased in both mouse strains after induction of allergic airway disease and localised in lung epithelial cells. Mice with targeted disruption of the Gpx-2 gene showed significantly enhanced airway inflammation compared to sensitised and challenged wild-type mice.

Our data indicate that genes encoding the antioxidants GPX2 and GSTO 1-1 are common inflammatory genes expressed upon induction of allergic airway inflammation, and independently of allergic susceptibility. Furthermore, we provide evidence to illustrate the importance of a single antioxidant enzyme, GPX2, in protection from allergen-induced disease.

KEYWORDS: Airway hyperreactivity, asthma, glutathione peroxidase, glutathione S-transferase

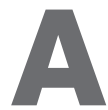
llergic asthma is a polygenetic disease that unfolds through the interplay of various genes with environmental factors. Despite the identification of several proteins and pathways involved in this inflammatory process, clinical trials in which key mediators were inhibited revealed that other, and as yet unknown, factors might be causally involved in the allergic cascade [1]. In search for these putative candidates, it is intriguing to speculate that antioxidant defence systems might be involved in the regulation of airway inflammation, since airways are naturally exposed to higher oxygen concentrations than most other tissues. Recent studies have identified increased levels of oxidative stress and alterations of antioxidant enzymes in the lungs of allergic individuals and in allergic animal models, resulting in the hypothesis that an increase in oxidative stress may contribute to the characteristic features of asthma [2].
Different technologies may be applied in search of new factors. Several recent studies benefitted from microarray analysis of gene expression profiles in order to identify genes involved in the development of allergic airway inflammation, as reviewed previously [3]. This approach has significant advantages over conventional experimental approaches. Conventional approaches only permit the study of known mediators of inflammation, where previous studies usually already suggested a possible association with allergic airway disease. Deductive gene expression profiling via microarrays, however, might identify mediators without any known link to inflammation or airway disease, thereby introducing truly "novel" targets into the field of allergic airway research. To identify novel factors commonly involved in pulmonary inflammation, we therefore employed RNA microarray technology. Comparisons of naïve and treated mice on the one hand, and of two mouse strains with known

\section{AFFILIATIONS}

*Dept of Paediatric Pneumology and Immunology, Charité Humboldt University,

"Dept of Internal Medicine/Infectious Diseases and Respiratory Medicine, Charité-Universitätsmedizin Berlin, Berlin,

\# Junior Research Group SFB 587

Hannover Medical School, Hannover,

${ }^{\S}$ University Children's Hospital, Ruhr University, Bochum, Germany.

${ }^{+}$Dept of Radiation Biology, Beckman Research Institute of City of Hope,

Duarte, CA, USA.

${ }^{\text {f}}$ Both authors contributed equally to this work.

\section{CORRESPONDENCE}

E. Hamelmann

University Children's Hospital

Bochum

Alexandrinenstraße 5

44791 Bochum

Germany

E-mail: eckard.hamelmann@

klinikum-bochum.de

Received:

Feb 202008

Accepted after revision:

Oct 082009

First published online:

Nov 062009 
different genetic susceptibilities to the induction of allergic airway disease [4] on the other hand, allowed us to identify common genes involved in pulmonary inflammation, independently from genetic susceptibility to disease development. Among the identified genes were several genes involved in the regulation of oxidative stress, among these the antioxidative enzymes, glutathione peroxidase-2 (GPX2) and glutathione $S$ transferase omega 1-1 (GSTO 1-1). These two enzymes had not previously been recognised to be part of the allergen-mediated inflammation cascade. Our data indicate that GSTO 1-1 and GPX2 are upregulated in allergic airway inflammation. Furthermore, the absence of GPX2 leads to an increase in the allergic airway inflammation. Manipulating this pathway in future studies will test the hypotheses that oxidative stress is involved in the pathogenesis of asthma.

\section{METHODS}

\section{Animals}

Specific-pathogen-free female BALB/c and C57BL/6 mice (Harlan-Winkelmann, Borchen, Germany), and C57BL/ 6 mice with a targeted disruption of Gpx-2 [5], 6-8 weeks old at the starting point of experiments, were used. Five animals per group were analysed and three independent experiments were conducted. All experimental procedures were approved by the animal care facility (Berlin Office for Occupational Safety, Protection of Health and Technical Safety-LAGeSo, Berlin, Germany).

\section{Sensitisation and challenge protocol}

Mice were sensitised by intraperitoneal injection of $20 \mu \mathrm{g}$ ovalbumin (OVA) grade VI (Sigma-Aldrich, Munich, Germany) in $2 \mathrm{mg}$ of aluminium hydroxide on days 1 and 14. Airway inflammation was induced by intranasal instillation of OVA grade V (Sigma-Aldrich) $(50 \mu \mathrm{g}$ in $50 \mu \mathrm{L}$ PBS) on day 28 (for microarray analyses) and 29 (for quantitative real-time RT-PCR). For studies with GPX2-null animals, mice were systemically sensitised by i.p. injection of OVA and aluminium hydroxide. Nonsensitised mice received aluminium hydroxide without OVA. On days 28, 29 and 30, all mice were challenged with OVA, and killed at day 32 . For microarray analyses, animals were sacrificed $16 \mathrm{~h}$ after the single intranasal challenge on day 28. For RT-PCR, western blotting and for studies with GPX2-null animals, animals were sacrificed $48 \mathrm{~h}$ after last challenge, i.e. either $48 \mathrm{~h}$ after two challenges on day 28 and 29 or 48 h after challenges on day 28,29 and 30.

\section{Detection of the allergic phenotype}

Immunoglobulins

$48 \mathrm{~h}$ after the last challenge, blood was drawn from the tail vein, and serum levels of total immunoglobulin (Ig)E and OVA-specific IgE were measured by ELISA, as previously described [6].

\section{Bronchoalveolar lavage}

$16 \mathrm{~h}$ after a single allergen challenge and $48 \mathrm{~h}$ after multiple challenges (day 28 and 29 or days 28, 29 and 30; see previous sections), lungs were lavaged and cytospin slides were prepared, stained with Diff-Quik (Dade Behring AG, Liederbach, Switzerland) and 200 cells were characterised according to morphological criteria via light microscopy.

\section{Airway reactivity}

Airway reactivity was measured by whole body barometric plethysmography (WBP; corresponding to the Buxco-system provided by EMKA Technologies, Paris, France), as previously described [6].

Invasive lung function measurement in isolated perfused mouse lungs after three challenges (days 28, 29 and 30) of wild-type and knockout mice: mouse lungs were prepared, ventilated and analysed as described [7]. After a steady state period of $30 \mathrm{~min}$, methacholine was administered to the perfusate for $30 \mathrm{~s}$ at 12-min intervals. Airway resistance values were determined at the end of the steady state period, as well as at the maximum level of resistance increase. The change in airway resistance was expressed as "relative fold airway resistance" ${ }^{\prime \prime}$, representing the increase in responsiveness due to OVA sensitisation by normalising fold airway resistance values of OVA lungs to corresponding mean values of control groups.

\section{Preparation of RNA}

Total RNA was extracted from mouse lungs using Qiagen RNeasy Total RNA isolation kit (Qiagen, Hilden, Germany) according to the manufacturer's instructions.

\section{Microarray analysis}

cRNAs were hybridised individually to mouse genome MGU74Av2 chips (Affymetrix, High Wycombe, UK). In total, eight lungs were analysed, two treated mice versus two controls in two different mouse strains (BALB/c and C57BL/6), respectively. The gene chips were scanned with an Affymetrix Gene Chip Instrument and scaled using Affymetrix's Microarray Suite software 5.0 (MAS5). We made four-way comparison of the arrays; between two different mouse strains and between treated and controls $(2 \times 2$ matrix $)$. Only those genes which were found to be similarly regulated in all four comparisons were classified as differentially expressed genes. The signal log ratio was converted to a standard on a logarithmic scale and the mean fold change of all four comparisons was calculated. We consecutively focused on those genes, which were similarly regulated in both strains of mice and with more than two-fold changes between treated and untreated mice. A more detailed description of the microarray analysis is attached as supplementary material.

\section{Real-time PCR}

PCR amplification and analysis were performed using an ABI PRISM 7700 (Perkin Elmer, Rodgau, Germany) and SDS software version 1.7 (a more detailed description of the real time PCR can be found in the online supplementary material).

\section{Primer design and sequences}

Complementary DNA PCR primers for amplification were designed using Primer3 Input software (Whitehead Institute for Biomedical Research, Cambridge, MA, USA) for DNA and RNA sequences obtained from GenBank, USA. The list of primers and sequences were archived as supplementary material.

\section{Protein preparation, SDS-PAGE and western blotting}

$1 \mathrm{~g}$ of mouse lung tissue (snap-frozen; stored at $-80^{\circ} \mathrm{C}$ ) was homogenised in digestion buffer. Aliquots of the lung homogenates were analysed via SDS-PAGE and western blotting using 
anti-GPX2 or anti- $\alpha$-GSTO antibodies (a detailed description is provided in the online supplementary material) [8,9].

\section{Immunohistochemistry}

Localisation of the GPX2 and GSTO 1-1 proteins was detected via immunohistochemistry using $4 \mu \mathrm{m}$ paraffin sections of lung tissue. Antigen retrieval was performed by heating the tissue sections for $6 \mathrm{~min}$ in pre-heated Dako target retrieval solution (Dako, Hamburg, Germany), using a pressure cooker. For detection of GSTO 1-1, the rabbit antiserum was diluted 10,000-fold [9] and detection of GPX2 was performed with a rabbit polyclonal anti-GPX2 antibody [8]. Biotinylated secondary anti-rabbit antibodies were used at dilution of 1:10,000 (Amersham Pharmacia Biotech, Freiburg, Germany). For signal amplification and visualisation of anti-GSTO 1-1 and antiGPX2, a tyramine amplification system (CSA kit; Dako) was used. As chromogen for the peroxidase-reaction, 3,3'-diaminobenzidine tetrahydrochloride (Dako) was used.

\section{Statistical analysis}

Data pertaining to the allergic phenotype was analysed statistically with the Mann-Whitney U-Test. Microarray analysis was done with MAS5 using a nonparametric statistical test (Wilcoxon signed rank test).

\section{RESULTS}

\section{Analysis of the allergic phenotype}

Systemic sensitisation with the allergen OVA in BALB/c mice leads to a significant increase in both total and OVA-specific IgE compared with animals that received only PBS (total $\operatorname{IgE}$ $1,639 \mathrm{ng} \cdot \mathrm{mL}^{-1}$ versus $924 \mathrm{ng} \cdot \mathrm{mL}^{-1}$; OVA-specific $\mathrm{IgE} 423 \mathrm{light}$ units $(\mathrm{LU}) \cdot \mathrm{mL}^{-1}$ versus $<6.2 \mathrm{LU} \cdot \mathrm{mL}^{-1}$ ). The inflammatory reaction in the airways showed a specific and time-dependent pattern for the different cell types in the bronchoalveolar lavage (BAL) fluid (see online supplementary table 1). $16 \mathrm{~h}$ after the single airway allergen challenge mostly neutrophils, but virtually no eosinophils, were detected. At this time point, airway hyperreactivity (AHR), measured via WBP [6] had not yet developed (data not shown). At a later time point (48 h), the BAL contained a robust eosinophilic and lymphocytic infiltration, corresponding to development of in vivo AHR.

\section{Identification of upregulated inflammatory genes in lung tissues of sensitised and challenged animals}

RNA isolated from whole lung tissue was used to generate Affymetrix-based gene expression profiles. Lung tissue was obtained at $16 \mathrm{~h}$ after a single allergen airway challenge to analyse genes at an early time point of airway inflammation in order to identify genes involved in the development of the characteristic T-helper type 2 phenotype of this model. Gene expression was compared between BALB/c mice and C57BL/6 mice because of their known differences in the development of airway inflammation and AHR [4]. While both strains develop significant airway inflammation, AHR and systemic sensitisation parameters such as allergen-specific IgE are much more pronounced in the BALB/c strain. We postulated that the comparison of these two strains would strengthen our aim ("to identify signature genes of airway inflammation") considerably compared to an approach utilising only one mouse strain, increasing the probability of identifying genes truly relevant in the development of allergic airway inflammation.
OVA-sensitised and OVA-challenged C57BL/6 mice had an altered expression of 370 probe sets compared to the naïve control mice, whereas OVA-challenged BALB/c mice had 2,128 probe sets changed in their expression levels. Between these two sets, 95 probe sets were consistently upregulated in both sensitised and challenged BALB/c and C57BL/6 mice, but only 31 probe sets coding for 27 different genes were upregulated at least two-fold in both mouse strains (the list of upregulated genes after OVA challenge is provided in the online supplementary material as table II). Gene ontogeny analysis revealed that among these common inflammatory genes, several were involved in response to oxidative stress. Two of them, GPX2 and GSTO 1, had not yet been reported in the context of allergic airway reaction, and were thus analysed further.

\section{Upregulation of GPX2 and GSTO 1-1 in allergen-induced airway inflammation is confirmed by quantitative $R T-P C R$} Quantitative RT-PCR was used to confirm the gene chip result. Sensitised and challenged mice (OVA/OVA) showed about two- and five-fold higher levels of GPX2 and GSTO 1-1 mRNA in lung tissue compared with animals in which airway inflammation was not induced (PBS/PBS) (fig. 1). Although the upregulation of GPX2 (fig. 1a) and GSTO 1-1 (fig. 1b) was found in both mouse strains after induction of allergic airway inflammation, in BALB/c mice the increase was even higher, as determined by the relative difference in fluorescence intensity between the target mRNAs and $\beta$-actin mRNA, a housekeeping gene.

\section{GPX2 and GSTO 1-1 proteins are expressed at higher levels in mice with allergic airway inflammation}

Western blotting with specific anti-GPX2 and anti-GSTO 1-1 antibodies was used to verify that an upregulation in mRNA levels leads to an increase in tissue protein levels of these enzymes in mice with allergic airway inflammation. Elevated expression levels of both proteins were detected in the lung tissue of mice challenged with OVA (OVA/OVA) as compared to PBS-treated control animals (PBS/PBS) (fig. 2). While these values attained statistical significance for GPX2 protein expression levels (fig. 2a), comparison of OVA-challenged mice to PBS controls revealed only trends towards higher expression levels for GSTO 1-1.

\section{Expression pattern of GPX2 and GSTO 1 in mouse lung}

Immunohistochemistry for GPX2 and GSTO 1-1 revealed distinct expression patterns for these proteins in mouse bronchial epithelium (fig. 3). Expression patterns of both proteins were similar in the lungs of untreated animals as well as in sensitised and challenged animals with regards to localisation. GPX2 expression, which so far had not been detected in the lung on a protein level, was found in basal cells (arrowheads; fig. 3a), revealing a pattern compatible with expression in the cells responsible for epithelial regeneration. GSTO 1-1 was found mainly in the apical parts of epithelial cells, sometimes appearing to be "budding" from the surface of the cells (arrowheads; fig. 3b), but secreted proteins were never detected by immunohistology inside the airway lumen.

\section{GPX2 protects against airway inflammation}

In order to evaluate the biological significance of our findings, we evaluated the consequences of GPX2 absence in the context 

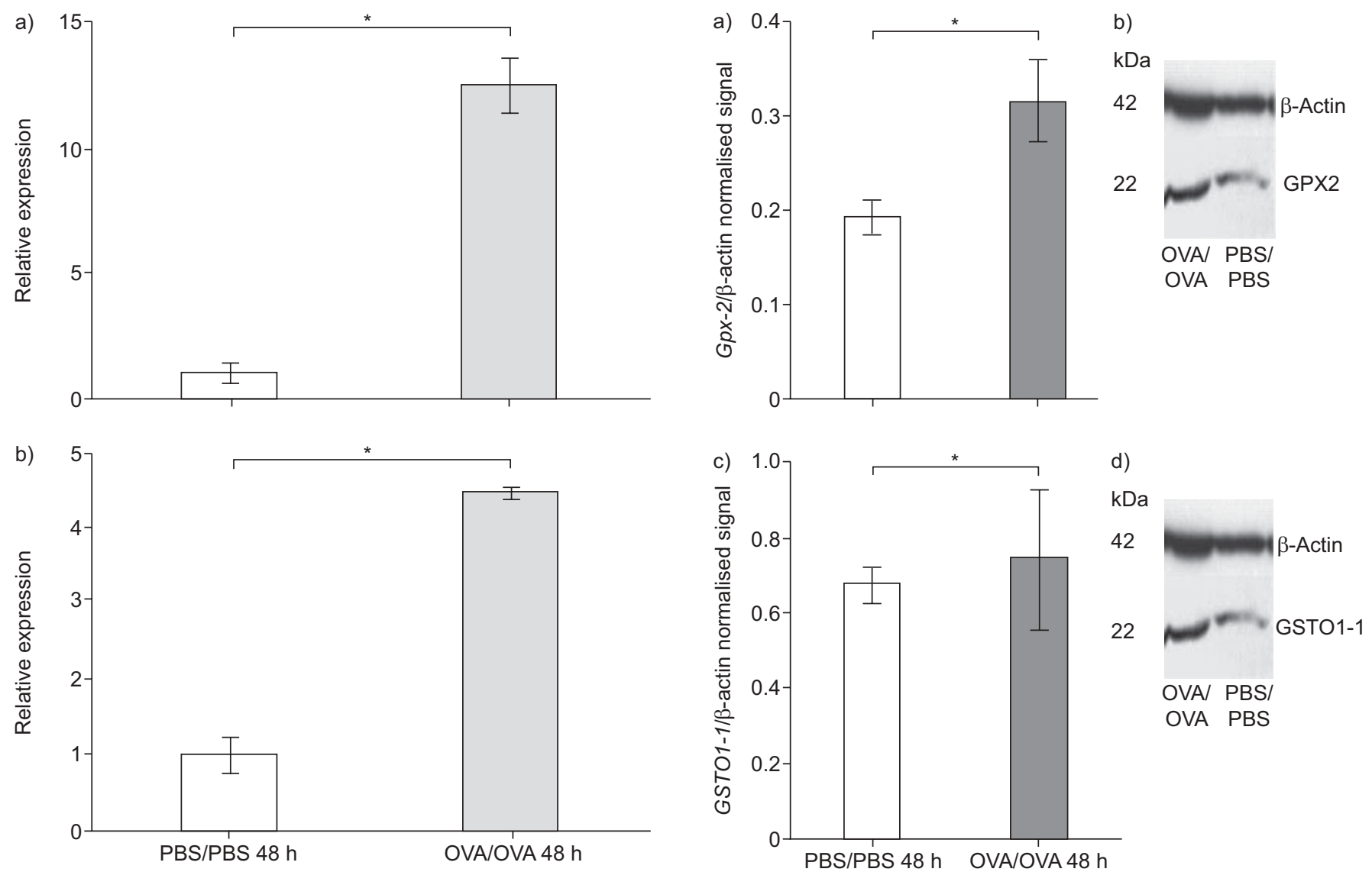

FIGURE 1. Glutathione peroxidase-2 (GPX2) and glutathione S-transferase omega 1-1 (GSTO 1-1) mRNA levels in the murine lung. mRNA levels of a) GPX2 gene and b) GSTO-1 gene were determined at $48 \mathrm{~h}$ after last challenge from mice subjected to ovalbumin (OVA) sensitisation and challenge (OVA/OVA) versus control mice (PBS/PBS) by real-time PCR. mRNA levels were initially normalised to $\beta$-actin mRNA levels. Comparisons were made by setting the value of control mice to one. Significance of mRNA expression was calculated via $\Delta \Delta C T$-method. *: $p \leqslant 0.05$, Mann-Whitney U-test.

of acute allergen-induced airway inflammation by utilising mice genetically deficient for GPX2 expression. As shown in figure 4, direct comparison of GPX2 knockout mice with wildtype littermates revealed significantly higher levels of airway inflammation in GPX2 knockout mice, mainly due to significant increased number of lymphocytes and eosinophils (fig. 4a). OVA-specific total $\operatorname{IgE}$ and $\operatorname{IgG}_{1}$ levels were also increased in GPX2 knockout mice but on a nonsignificant level (fig. $4 \mathrm{~b}$ and c). In order to evaluate functional consequences of GPX2 deficiency, we analysed airway resistance after methacholine provocation in isolated and perfused lungs from wildtype and knockout mice. Here, we observed a $32 \%$ increase in relative fold airway resistance in knockout mice in comparison to wild-type mice (fig. $4 \mathrm{~d}$ ).

\section{DISCUSSION}

In the present study, we utilised gene expression profiling in lung tissues of two different mouse strains to identify novel and common inflammatory genes involved in allergic airway disease. We detected two antioxidants, GSTO 1-1 and GPX2, which had yet not been recognised in this context and which

FIGURE 2. Glutathione peroxidase-2 (GPX2) and glutathione S-transferase omega 1-1 (GSTO 1-1) protein levels in murine lungs. Relative quantity of a) GPX2 or c) GSTO 1-1 protein levels were compared to protein levels of $\beta$-actin using integrated density values from western blot analyses ( $b$ and $d$ ) $48 \mathrm{~h}$ after last challenge. OVA: ovalbumin. *: $p \leqslant 0.05$, Mann-Whitney U-test.

were significantly upregulated, both on the transcriptional and translational levels. Our data support recent evidence that chronic allergic airway inflammation is, in part, a result of and mediated by reactive oxygen species (ROS) [2]. Furthermore, increased levels of inflammation and airway reactivity in GPX2-null mice support the notion that GPX2 plays a protective role in airway inflammation, similar to its antiinflammatory role in the gastrointestinal tract [5].

The GPX family consists of four selenoproteins, GPX1-4, which are key enzymes in the redox cycle. Their differential expression patterns and additional enzymatic capacities indicate that they play an important role in exerting cell- and tissue-specific roles in metabolic regulation [10]. GPX1-4 have all been reported to be expressed in human lungs [11], yet functional studies revealing their contributions to health and disease in this organ remain sparse. HOFFMANN et al. [12] have recently shown that GPX1, but not GPX4 protein was elevated (2.8-fold) in lung tissues of challenged C57BL/6J mice analysed on day 29. In our analysis, we were not able to reproduce this increase. However, induction of GPX1 gene expression might occur later in the time course of allergic inflammation than the time point analysed in our study. 

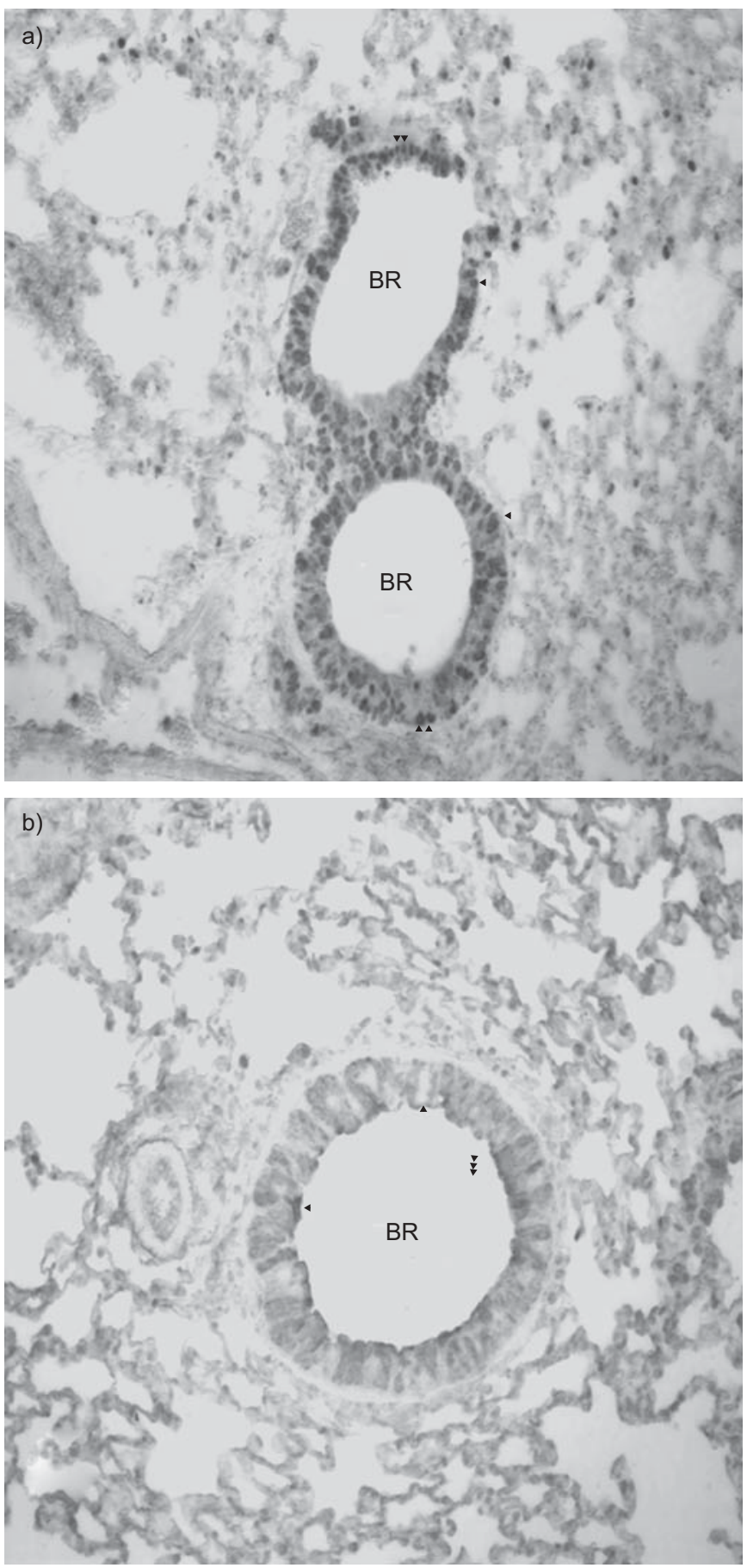

FIGURE 3. Localisation of glutathione peroxidase-2 (GPX2) and glutathione $S$-transferase omega 1-1 (GSTO 1-1) in murine lungs. Immunohistochemical detection of a) GPX2 and b) GSTO 1-1 protein expression in murine lungs. The GSTO 1-1 protein was found mainly in apical parts of epithelial cells (arrowheads) while the GPX2 protein was localised in basal epithelial cells (arrowheads). Protein expression was revealed via immunohistochemistry of paraffin cuts in lungs harvested $48 \mathrm{~h}$ after challenge. BR: bronchus.

Most studies of GPX2 so far were confined to the gastrointestinal tract $[5,13,14]$. Although mRNA GPX2 expression was found in mouse lungs, localisation in this organ has not been elucidated [15]. In humans, GPX2 protein expression has
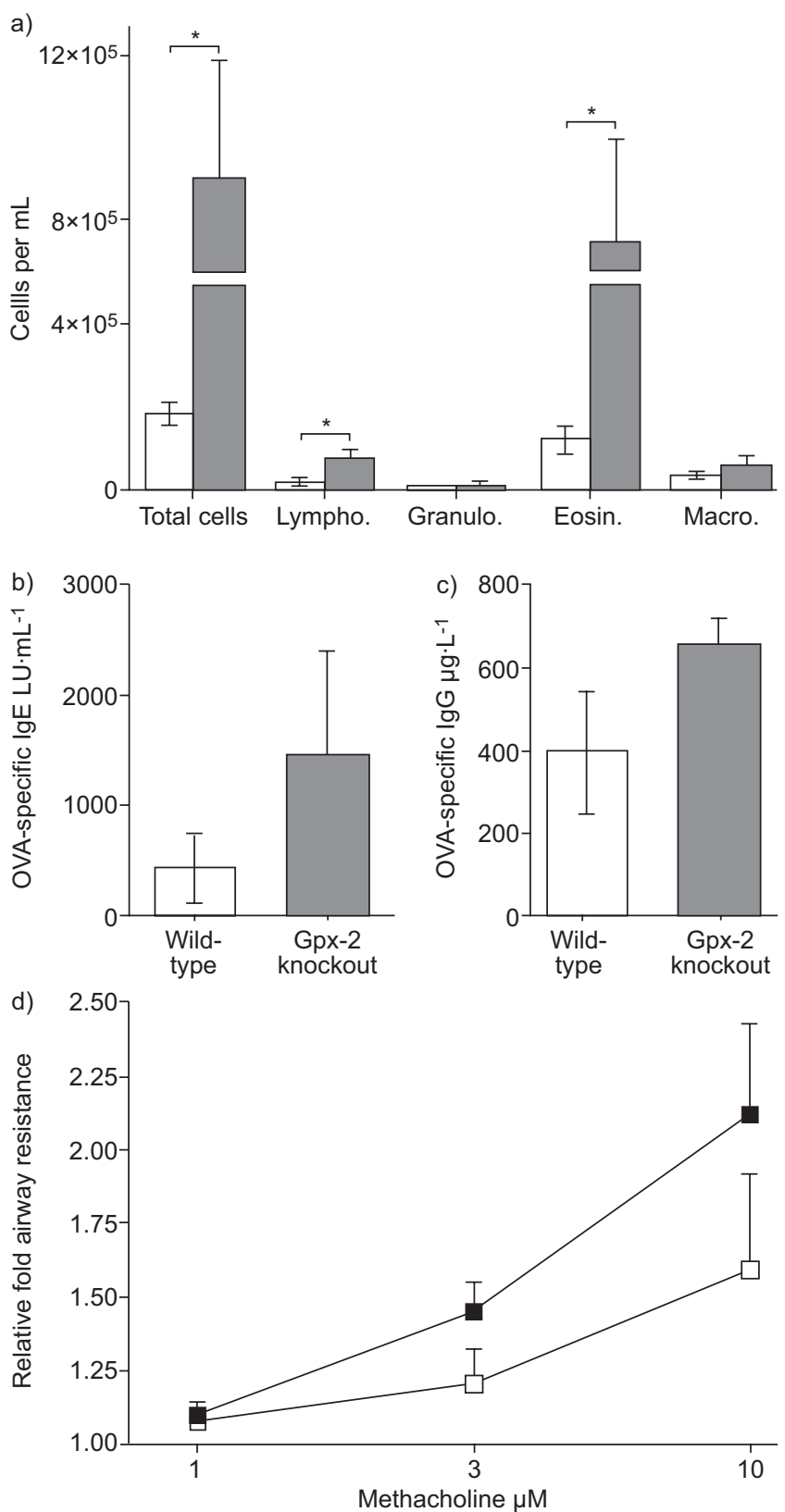

FIGURE 4. Glutathione peroxidase-2 (GPX2) deficiency enhances allergic airway inflammation and airway reactivity. a) Compared with wild-type littermates $(\square ; n=6)$, GPX2 knockout mice ( $\square$; n=6) showed increased airway inflammation due to an increased influx of eosinophils (eosin.) and lymphocytes (lympho.) after sensitisation and challenge with antigen. Ovalbumin (OVA)-specific immunoglobulin $(\mathrm{Ig}) \mathrm{E}$ (b) and $\lg \mathrm{G}_{1}$ (c) were increased in GPX2 knockout mice but not to a significant level. d) Comparing airway resistance in isolated perfused lungs of GPX2 knockout ( $\mathbf{\square})$ and wild-type $(\square)$ mice ( $\mathrm{n}=6$ each) after sensitisation and challenge, we found a higher relative fold airway resistance in GPX2 knockout mice than in the corresponding wild-type controls. Granulo.: granulocytes; macro.: macrophages. *: $p<0.05$

not been detected in the lung [13] and GPX activity in the lung has mainly been attributed to GPX1 activity [16].

GPX2 is upregulated by nuclear factor erythroid 2-related factor 2 (Nrf2), and increased levels in hyperoxia-induced lung 
injury in Nrf2-null mice points towards a role of GPX2 in protection against oxidative stress $[15,17]$. The present study extends these findings to another adverse lung event that is known to generate ROS as well as inflammatory mediators: allergic airway inflammation. The finding of increased airway inflammation in the absence of GPX2 strongly supported a protective role in allergic airway inflammation for this protein.

Considering that Ho et al. [16] have shown that $95 \%$ of the glutathione peroxidases activity in the lung is attributable to GPX1, it might be possible that the protective role of GPX2 in this organ is due to different enzymatic activities. In fact, glutathione peroxidases have been reported to inhibit prostaglandin synthesis [18], thus reducing the expression of proinflammatory mediators known to play an important role in the pathogenesis of allergic asthma. Furthermore, GPX2 knockout studies have suggested an involvement in antiinflammatory mechanisms [5]. The exact mechanism by which GPX2 decreases airway inflammation will be the subject of further studies as our preliminary studies concerning changes in classical inflammatory mediators (interleukin (IL)-4, IL-5, IL-10 and interferon- $\gamma$ ) upon allergen re-stimulation did not show significant differences (data not shown).

The other gene identified in our present study belongs to the supergene family of glutathione $S$-transferases, of which we identified the mouse homologue of glutathione $S$-transferase omega 1-1 (GSTO 1-1). In the human lung, the enzyme GSTO 1-1 is reported to be exclusively expressed in alveolar macrophages [19] while the mouse homologue has been shown to be expressed ubiquitously, with expression levels highest in the lung and liver [9].

Until now, this enzyme has not been implicated in the pathology of bronchial asthma. The glutathione $S$-transferase family was initially described to provide an important detoxification step for various ROS [20] but many of the six distinct subclasses perform additional reactions [21]. Human GSTO 1-1 acts as a glutathione-dependent thioltransferase, which might serve to restore enzymatic function after exposure to oxidative stress [22]. Human GSTO 1-1 has also been shown to inhibit IL-1 $\beta$-dependent apoptosis via cytokine release inhibitory drugs [23], suggesting a new type of regulatory operation performed by this enzyme. The expression of the mouse homologue of GSTO 1-1, p28, was initially discovered in a radiation-resistant lymphoma line, pointing towards a possible role in conferring resistance to radiation-induced cell death [9]. This role is supported by studies showing that glutathione $S$-transferases inhibit certain stress kinases, such as Jun N-terminal kinase [24], which in turn inhibits apoptosis and allows cell repair [25]

Integrating the functional results pertaining to GSTO 1-1 into our mouse model, inhibition of apoptosis by upregulation of GSTO 1-1 may lead to adverse effects in cells that, under normal circumstances, would be deleted. One hypothesis concerning the effects of ROS proposes three levels of response to oxidative stress: 1) low amounts of oxidative stress induce protective responses via the induction of cytoprotective and anti-inflammatory mediators; 2) an intermediate level of oxidative stress causes the induction of cytokines, chemokines and adhesion molecules, leading to an inflammatory response; and, finally, 3) a high amount of oxidative stress causes apoptosis and necrosis, which leads to the induction of inflammation and remodelling in which induction of GSTO 1-1 might play a role, as reviewed previously [25]

Other findings pointing towards a possible role of GSTO 1-1 and GPX2 in allergic airway diseases arise from studies on genetic heterogeneity. The individual's ability to deal with an oxidant burden may depend in part on genetic background. Polymorphisms of different subclasses of glutathione $S$ transferases have been shown to be associated with asthma, lung function and susceptibility to xenobiotic enhancement of allergic symptoms [21, 26, 27]. Recently, functional data have been added, suggesting that glutathione $S$-transferases are able to modify the adjuvant effect of diesel exhaust particles and thereby attenuate local and systemic allergic inflammation [26]. Such an association has not yet been reported for the GSTO 1-1 or the GPX2 isoenzyme. Yet two independent studies were able to link the development of asthma to chromosome 14q24, which is the chromosomal location of GPX2 [28, 29]. Taken together, our data suggest that different activity levels of GSTO 1-1 and GPX2 due to genetic polymorphisms might contribute to the relative risk of disease development, a hypothesis that should be tested in association studies in disease cohorts.

In summary, we have identified two common inflammatory genes that were not previously recognised as being involved in the development of allergen-mediated airway disease. Knowledge of the mechanism underlying oxidative stress in the lungs may allow the development of novel antioxidant interventions. These strategies will then have to test the hypothesis that oxidative stress is involved in the pathogenesis of asthma, not only by direct injury to cells, but also as a fundamental factor in airway inflammation.

\section{SUPPORT STATEMENT}

This work was supported by grants from the German National Genome Research Network (Federal Ministry of Research (BMBF) 01GS0120) (E. Hamelmann), the Crohn's and Colitis Foundation of America (F-F. Chu) and National Institutes of Health (Bethesda, MD, USA) R01 CA114569 (F-F. Chu).

\section{STATEMENT OF INTEREST}

None declared.

\section{ACKNOWLEDGEMENTS}

We thank C. Seib (Charité Universitätsmedizin Berlin, Berlin, Germany) for her excellent technical assistance.

\section{REFERENCES}

1 Leckie MJ, ten Brinke A, Khan J, et al. Effects of an interleukin-5 blocking monoclonal antibody on eosinophils, airway hyperresponsiveness, and the late asthmatic response. Lancet 2000; 356: 2144-2148.

2 Bowler RP, Crapo JD. Oxidative stress in allergic respiratory diseases. J Allergy Clin Immunol 2002; 110: 349-356.

3 Dittrich AM, Quarcoo D, Krokowski M, et al. Gene expression profiling as novel tool in experimental asthma research. Exp Toxicol Pathol 2006; 57: Suppl. 2, 31-33.

4 Herz U, Braun A, Ruckert R, et al. Various immunological phenotypes are associated with increased airway responsiveness. Clin Exp Allergy 1998; 28: 625-634. 
5 Esworthy RS, Aranda R, Martin MG, et al. Mice with combined disruption of Gpx1 and Gpx2 genes have colitis. Am J Physiol Gastrointest Liver Physiol 2001; 281: G848-G855.

6 Hamelmann E, Vella AT, Oshiba A, et al. Allergic airway sensitization induces $\mathrm{T}$ cell activation but not airway hyperresponsiveness in B cell-deficient mice. Proc Natl Acad Sci USA 1997; 94: 1350-1355.

7 Witzenrath M, Ahrens B, Kube SM, et al. Detection of allergeninduced airway hyperresponsiveness in isolated mouse lungs. Am J Physiol Lung Cell Mol Physiol 2006; 291: L466-L472.

8 Chu FF, Esworthy RS, Akman S, et al. Modulation of glutathione peroxidase expression by selenium: effect on human MCF-7 breast cancer cell transfectants expressing a cellular glutathione peroxidase cDNA and doxorubicin-resistant MCF-7 cells. Nucleic Acids Res 1990; 18: 1531-1539.

9 Kodym R, Calkins P, Story M. The cloning and characterization of a new stress response protein. A mammalian member of a family of theta class glutathione s-transferase-like proteins. J Biol Chem 1999; 274: 5131-5137.

10 Brigelius-Flohe R. Tissue-specific functions of individual glutathione peroxidases. Free Radic Biol Med 1999; 27: 951-965.

11 Chu FF, Esworthy RS, Doroshow JH, et al. Expression of plasma glutathione peroxidase in human liver in addition to kidney, heart, lung, and breast in humans and rodents. Blood 1992; 79: 3233-3238.

12 Hoffmann PR, Jourdan-Le SC, Hoffmann FW, et al. A role for dietary selenium and selenoproteins in allergic airway inflammation. J Immunol 2007; 179: 3258-3267.

13 Chu FF, Doroshow JH, Esworthy RS. Expression, characterization, and tissue distribution of a new cellular selenium-dependent glutathione peroxidase, GSHPx-GI. J Biol Chem 1993; 268: 2571-2576.

14 Komatsu H, Okayasu I, Mitomi H, et al. Immunohistochemical detection of human gastrointestinal glutathione peroxidase in normal tissues and cultured cells with novel mouse monoclonal antibodies. J Histochem Cytochem 2001; 49: 759-766.

15 Singh A, Rangasamy T, Thimmulappa RK, et al. Glutathione peroxidase 2, the major cigarette smoke-inducible isoform of GPX in lungs, is regulated by Nrf2. Am J Respir Cell Mol Biol 2006; 35: 639-650.

16 Ho YS, Magnenat JL, Bronson RT, et al. Mice deficient in cellular glutathione peroxidase develop normally and show no increased sensitivity to hyperoxia. J Biol Chem 1997; 272: 16644-16651.
17 Cho HY, Jedlicka AE, Reddy SP, et al. Role of NRF2 in protection against hyperoxic lung injury in mice. Am J Respir Cell Mol Biol 2002; 26: 175-182.

18 Sakamoto H, Imai H, Nakagawa Y. Involvement of phospholipid hydroperoxide glutathione peroxidase in the modulation of prostaglandin D2 synthesis. J Biol Chem 2000; 275: 40028-40035.

19 Yin ZL, Dahlstrom JE, Le Couteur DG, et al. Immunohistochemistry of omega class glutathione S-transferase in human tissues. J Histochem Cytochem 2001; 49: 983-987.

20 Hayes JD, Strange RC. Potential contribution of the glutathione S-transferase supergene family to resistance to oxidative stress. Free Radic Res 1995; 22: 193-207.

21 Strange RC, Spiteri MA, Ramachandran S, et al. Glutathione-Stransferase family of enzymes. Mutat Res 2001; 482: 21-26.

22 Board PG, Coggan M, Chelvanayagam G, et al. Identification, characterization, and crystal structure of the Omega class glutathione transferases. J Biol Chem 2000; 275: 24798-24806.

23 Laliberte RE, Perregaux DG, Hoth LR, et al. Glutathione stransferase omega 1-1 is a target of cytokine release inhibitory drugs and may be responsible for their effect on interleukin-1 $\beta$ posttranslational processing. J Biol Chem 2003; 278: 16567-16578.

24 Adler V, Yin Z, Fuchs SY, et al. Regulation of JNK signaling by GSTp. EMBO J 1999; 18: 1321-1334.

25 Vignola AM, Chiappara G, Gagliardo R, et al. Apoptosis and airway inflammation in asthma. Apoptosis 2000; 5: 473-485.

26 Gilliland FD, Gauderman WJ, Vora $\mathrm{H}$, et al. Effects of glutathione-S-transferase M1, T1, and P1 on childhood lung function growth. Am J Respir Crit Care Med 2002; 166: 710-716.

27 Gilliland FD, Li YF, Saxon A, et al. Effect of glutathione-Stransferase M1 and P1 genotypes on xenobiotic enhancement of allergic responses: randomised, placebo-controlled crossover study. Lancet 2004; 363: 119-125.

28 Mansur AH, Bishop DT, Markham AF, et al. Suggestive evidence for genetic linkage between IgE phenotypes and chromosome 14q markers. Am J Respir Crit Care Med 1999; 159: 1796-1802.

29 Hakonarson H, Bjornsdottir US, Halapi E, et al. A major susceptibility gene for asthma maps to chromosome 14q24. Am J Hum Genet 2002; 71: 483-491. 